\title{
A Differentiation Based Framework to Multi-Objective Transmission Expansion Planning Considering Meteorological Disaster
}

\author{
Yunfei Mu ${ }^{\mathrm{a} *}$, Yuan Zeng ${ }^{\mathrm{a}}$, Hongjie Jia ${ }^{\mathrm{a}}$, Jinshan Luo ${ }^{\mathrm{b}}$, Xiaohui Wang ${ }^{\mathrm{b}}$ \\ ${ }^{a}$ Key Laboratory of Smart Grid of Ministry of Education, Tianjin University, Tianjin, 300072, China \\ ${ }^{b}$ State Power Economic Research Institute, Beijing, 100761, China
}

\begin{abstract}
The frequent occurrences of meteorological disasters (MD) have revealed the deficiencies of the transmission expansion planning (TEP). The traditional TEP methods fail to establish the mathematical relationship between the MD and the transmission component outage rate (TCOR) when calculating the outage cost of customer. As a result, the optimized schemes are unable to determine the minimum backbone grid that should be guaranteed in the event of MDs, which is against the basic principles of economy and reliability. In this paper, the research status of multiobjective TEP is reviewed and the mathematical relationship between MD and TCOR is investigated preliminarily. On this basis, a differentiation based framework to multi-objective TEP is proposed and discussed, which is of significant reference value to the TEP under the meteorological disaster-prone environment.
\end{abstract}

Keywords: Meteorological disaster, multi-objective transmission expansion planning, transmission component outage rate, differentiation, minimum backbone grid

\section{Introduction}

Multi-objective transmission expansion planning (TEP) is formulated as an optimization problem including two or more objectives with certain constraints. The purpose of TEP is to design an electricity transmission network as economical as possible while providing a reliability power supply. How to determine the cost-effective TEP scheme is one of the most challenging optimization problems of power system due to the mathematical formulation which leads to a complicated, integer-valued, non-convex, non-linear mathematical programming problem, especially when the system size is enlarged [1]-[5].

A difficulty in TEP is the coordination between the objectives of economy and reliability. The traditional TEP methods usually establish the multi-objective optimization model which takes the economy and reliability into account together by incorporating the minimum exploitation cost of supplier and the minimum outage cost of customer at the same time [6], [7]. However, such methods fail to establish the mathematical relationship between the meteorological disaster (MD) and the transmission component outage rate (TCOR) when calculating the outage cost of customer. Definitely, TCOR is a function of the meteorology where the transmission component lies. Once the extreme meteorology disaster happens, the surging increase of transmission outage rate may lead to large-scale blackouts of power system and result huge economic losses. For example, the ice storm in 1998 hit Canadian provinces of Ontario and Quebec and parts of the northeastern U.S. in January. This storm caused extensive power outages across the impacted regions and is generally acknowledged as Canada's costliest

* Manuscript received July 20, 2012; revised August 24, 2012.

Corresponding author. Tel.: +86-22-27409881-632; E-mail address: yunfeimu@tju.edu.cn.

This work is supported by State Grid Corporation of China, Major Projects on Planning and Operation Control of Large Scale Grid (SGCC-MPLG028-2012). 
MD. Due to the collapse of transmission lines and supporting structures from ice accumulation, over 4.7 million people in Canada and another 500,000 in the U.S. lost power during the storm. The Canadian utility company Hydro-Quebec was particularly badly hit. Over 3,000 km of Hydro-Quebec's power network was impacted by the ice storm, with more than 24,000 poles, 4,000 transformers, and 1,000 steel pylons in need of repair [8]. The similar ice storm happened in the southern China at the beginning of the year of 2008. Transmission lines and power towers were covered with thick ice and collapsed throughout large areas. By Feb. 26th, the freezing weather caused 7,541 power transmission lines with $10 \mathrm{kV}$ and above to fail. Over 859 substations providing $35 \mathrm{kV}$ and above were forced to be out of service. The power loss was 6,209 billion $\mathrm{kWh}$ and the affected population reached as high as 261.82 million [9].

The past lessons show that it is imperative to construct disaster resistance transmission network by improving the fortification standard of transmission components. However, it is not economical to strengthen all the transmission components for pursuing reliability. As a result, it is important to determine the minimum backbone grid that should be guaranteed in the event of MDs, which can satisfy the basic guidelines of economy and reliability. In this paper, the research status of multi-objective TEP is reviewed and the mathematical relationship between MD and TCOR is investigated preliminarily. On this basis, a differentiation based framework to multi-objective TEP considering MD is proposed to determine the critical transmission components for constituting the minimum backbone grid, which is of significant reference value to TEP under the meteorological disaster-prone environment.

\section{Review of Multi-Objective Transmission Expansion Planning}

\subsection{General optimization model of multi-objective TEP}

TEP is formulated as a multi-objective problem with a set of equality and inequality operational constraints. The general optimization model of multi-objective TEP is depicted as (1) [10].

$$
\begin{aligned}
& V-\min \mathbf{f}(\mathbf{x}) \\
& \text { s.t. } \mathbf{X}=\left\{\begin{array}{ll}
\mathbf{x} \in R^{n} \mid \begin{array}{l}
g_{j}(\mathbf{x}) \geq 0, j=1,2, \cdots, p \\
h_{k}(\mathbf{x})=0, k=1,2, \cdots, q
\end{array}
\end{array}\right\}
\end{aligned}
$$

where $\mathbf{x}=\left[x_{1}, x_{2}, \ldots, x_{n}\right]$ is the vector of decision variable; $\mathbf{X}$ is the feasible region or constraint set of the model; $g_{j}(\mathbf{x})$ and $h_{k}(\mathbf{x})$ are the inequality and equality constraints; $f(\mathbf{x})=\left[f_{1}(\mathbf{x}), f_{2}(\mathbf{x}), \ldots, f_{m}(\mathbf{x})\right]^{\mathrm{T}}$ is the vector of objective function. $\mathrm{V}$-min denotes the vector minimization, which means each objective in the objective function is minimized equivalently.

- Objective function

In order to address the various needs of modern TEP, the objective functions of multi-objective TEP are flexible. Usually, the objective functions depicted in (1) can be classified into two groups: economy and reliability [10-12]. For example, the minimization of consturction cost of new facilities, the minimization of transimission tariffs, the minimization of system operational cost (maintenance and energy loss), and the minimization of land use, etc., belong to the group of economy; while the outage cost of customer, the maximization of transmission adequacy (security), etc., can be categorized into reliability. However, the deregulation of power system has introduced new objectives and goals in the field of TEP, such as the minimization of congestion cost, the maximization of transimision capability, the maximiaztion of transient stabiltiy and the minimization of environmental impacts, etc.

The above objective functions have different levels of importance and even contradict with each other. The pursuit of high reliability will lead to the decreasing of economy. On the contrary, too much emphasis on the economy will in turn weaken the electrical interconnections of grid, causing the decline of reliability. The general approach to tackle this problem is to incorporate the minimum exploitation cost of supplier and the minimum outage cost of customer at the same time. Evidently, the outage cost is a function of TCOR. The existing methods to determine TCOR only take the general failures into 
considersation but fail to evaluate the significant impacts from the meteorological disaster which happens more and more frequently than ever before.

- Constraints

The TEP should not only meet the demand increase and all types of electricity transactions but comply with the power supply quality and security standards $[1-5,10]$. A large number of constraints exist in the TEP. In addition to the equality constraints of power flow, there are also inequality constraints, such as the thermal limits of transmission lines, the capital investment limits, the maximum acceptable power loss limits, the maximum acceptable value of system expected demand not served limits, the minimum acceptable value of new-added right-of-way occupied area limits, the voltage and frequency limits, the static and dynamic stability limits, etc. In practical engineering applications, the above constraints are always simplified and only the static constraint of ' $N-k$ ' is considered.

- Decision variable

The basic decision variable in TEP is a given set of transmission lines for selecting [13]. The integer value of each transmission line determines whether the line is selected or not and the corresponding fortification standard. Also these integer values can reflect the topology of transmission network under planning with its robustness. Besides, the flexible AC transmission system (FACTS), with the advantages of increasing transmission capacity, reducing the investment cost, dynamic power flow control, and increasing static and dynamic stability, becomes a new kind of decision variable [14]. Also, with the development of demand response, the electricity price is another kind of important decision variable [15].

However, the traditional TEP methods fail to consider the differentiations of each transmission line, such as the level of importance, the location and environment, etc., which only implement the established standards according the voltage levels at the construction stage. As a result, these kinds of methods cannot evaluate the economical and reliable objectives comprehensively, which is difficult in satisfying the reliability index, especially in the MD-prone environment.

\subsection{Solution approach}

The multi-objective TEP has been defined as a complex combinatorial optimization problem with conflicting, non-conflicting and uncertain relationships. Generally, there are two general approaches to solve the multiple objectives problem. In the first place, the multiple objective functions are reduced into a single function, which is called the weighted sum of each objective $[6,10,11,16]$. The compounded objective is then optimized by means of the single objective function to obtain the unique optimal solution. The classical methods in this aspect are: weighted aggregation, hierarchical optimization method, fuzzy set theory, fuzzy set pair analysis and optimal deviation method, etc. However, due to the difficulties in coordinating the dimension, weights and their relationships, the above methods will inevitably cause approximation among various objectives.

The Pareto based methods tackle this problem from a new perspective, which can effectively compensate for the deficiencies of the single objective based methods [2]. The essence of Pareto based method is the search of the optimal Pareto Set in which every Pareto-optimal point is an equally acceptable solution for the multi-objective optimization and the decision is left for the planner. Several intelligent and heuristics methods are contributing to calculate the optimal Pareto Set, such as the Genetic Algorithm [17], Simulated Annealing [18], Particle Swarm Optimization [19], Tabu Search and Ant Colony Algorithm, etc. [20, 21].

\section{Multi-Objective Transmission Expansion Planning Considering Meteorological Disasters}

The frequently happened MDs bring significant challenges to the reliable operation of transmission grid. As depicted in Section 2, the existing multi-objective TEP methods are unable to determine the minimum backbone grid that should be guaranteed in the event of MDs, which is against the basic guidelines of economy and reliability. As a result, it is critical to determine the weak parts of the 
transmission network based the concept of differentiation. In this paper, the static TEP is used for illustration purpose $[5,10]$.

\subsection{Calculation of the outage cost of customer}

Among various reliability indexes of TEP, only the indexes which can be transformed into the economical based indictor are able to be treated as the objective function. For this reason, the exploitation cost of supplier and the outage cost of customer which has closely relationship with TCOR are used as the objective functions in this paper. The outage cost of customer $C_{\text {out }}$ is depicted as (2) [6].

$$
C_{\text {out }}=8760 \eta \sum_{i \in \mathbf{\Omega}}\left(L_{i} p_{i}\right)
$$

where $\eta$ is the outage cost per unit of electricity; $L_{i}$ is the load reduction under the condition of fault $i ; p_{i}$ is the occurrence probability of fault $i$; $\Omega$ is the fault set.

The $p_{i}$ has closely relationship with TCOR. In order to calculate the TCOR accurately, thus the fault set $\Omega$, Poisson distribution representing the characteristics of discrete and simple calculation is used to describe the uncertain events which occur in a fixed time period with a known average rate and independent of time [22]. Poisson distribution expresses the expected number of transmission component failure event which occurs in unit time interval is $\lambda$. The probability function $H(k)$ of Poisson distribution that there are exactly $k$ occurrences is depicted in (3).

$$
H(k)=\frac{\lambda^{k}}{k !} e^{-\lambda} \quad(k=0,1,2, \ldots, K)
$$

For a TEP scheme, the state of the transmission network is expressed by

$$
\mathbf{s}=\left(s_{1}, s_{2}, \ldots, s_{t}\right)
$$

where $\mathbf{s}$ is the system state vector which depicts the state combination of the $t$ transmission lines; $s_{k}$ is the state of line $k$, where $s_{k}=1$ means the line is under normal operation and $s_{k}=0$ means the line is out of service due to the fault. $s_{k}$ is determined by $H(k)$.

IEEE standard 348 suggests that the weather environment should be divided into three classes: normal, adverse and major storm disaster conditions [23]. A three-state weather model corresponding to these three classes are used to incorporate the weather conditions into the reliability analysis in [24]. Based on the three-state weather model, the decision variables used in this paper are the states of transmission lines, which are listed in Table I. $\lambda$ is a key parameter to determine $H(k)$, thus $\boldsymbol{s}$ of the TEP scheme. The larger of $\lambda$, the more faults on transmission lines. The $\lambda$ used for the three fortification standard levels are listed in Table I with $\lambda_{\min }<\lambda_{\text {mid }}<\lambda_{\max }$, which can be determined by the statistical analysis on historical weather data.

Table 1. Decision variables used in TEP considering MD

\begin{tabular}{lccccc}
\hline & \multicolumn{2}{c}{ Construction or not } & \multicolumn{3}{c}{ Fortification standard level } \\
\hline Weather conditions & Not Construction & Construction & Level I & Level II & Level III \\
\hline Normal & $\sqrt{ }$ & $\sqrt{ }$ & - & - & $\sqrt{ }$ \\
Adverse & $\sqrt{ }$ & $\sqrt{ }$ & - & $\sqrt{ }$ & $\sqrt{ }$ \\
Major storm disaster & $\sqrt{ }$ & - & Very high & High & Low \\
Cost of construction & - & - & Very high & High & Low \\
Reliability & - & - & $\lambda_{\text {min }}$ & $\lambda_{\text {mid }}$ & $\lambda_{\text {max }}$ \\
Value of $\lambda$ & - & & &
\end{tabular}

Then the outage cost of customer is obtained by the Monte Carlo process. Using $H(k)$ with the different levels of $\lambda$ to determine a system state vector $\boldsymbol{s}$. This process is repeated $N$ times to generate the 
fault set $\boldsymbol{\Omega}$ which is composed of $N$ system state vectors, where $\boldsymbol{\Omega}=\left(\mathbf{s}_{1}, \mathbf{s}_{2}, \ldots, \mathbf{s}_{N}\right)$. The control algorithm function $F(\mathbf{s})$ is used to determine the load reduction under each system state when fault happens. The load reduction expectation in (2) is obtained by (5), which can be used to determine the outage cost of customer.

$$
\sum_{i \in \Omega}\left(L_{i} p_{i}\right)=\frac{1}{N} \sum_{j=1}^{N} F\left(\mathbf{s}_{j}\right)
$$

\subsection{Model and framework of multi-objective TEP considering MDs}

The objective functions of the differentiation based multi-objective TEP are depicted in (6) (7). The corresponding constraints are given in (8) (9) and the " $N$-1" standard.

$$
\begin{aligned}
& \min f_{1}=C_{\text {out }} \\
& \min f_{2}=M \sum_{i=1}^{t} K_{i} l_{i} \\
& \text { s.t. } \mathbf{B} \boldsymbol{\theta}=\mathbf{P} \\
& \mathbf{P}_{L} \leq \mathbf{P}_{L \text {.max }}
\end{aligned}
$$

where $f_{2}$ is the exploitation cost of supplier; $M$ is the capital recovery factor; $K_{i}$ is the cost of transmission line $i$ per length unit; $l_{i}$ is the length of transmission line $i$ under construction; $\mathbf{B}$ is the admittance matrix of system; $\mathbf{P}$ is the node power injection vector; $\mathbf{P}_{L}$ and $\mathbf{P}_{L \text {, max }}$ are the vectors of power flow and the maximum power flow of transmission line. The Pareto based intelligent approaches can be used to solve the above optimization problem.

The framework of the differentiation based multi-objective TEP considering MDs is shown in Fig. 1.

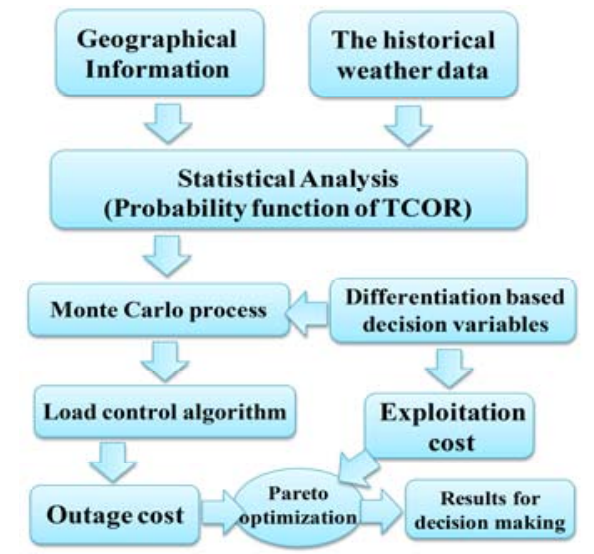

Fig. 1. Framework of the differentiation based multi-objective TEP considering MDs

\section{Conclusion}

In this paper, a differentiation based framework to multi-objective TEP considering MDs is proposed and discussed. According to the geographical information and historical weather data, the statistical analysis is used to obtain the probability functions of TCOR under different differentiation based decision variables (construction and standard levels). Then, Monte Carlo process and load control algorithm are introduced to determine the outage cost of customer. The outage cost of customer is incorporated with the exploitation cost of supplier as the objective functions of the multi-objective TEP. Various Pareto based intelligent optimization approaches can be used to obtain the optimal Pareto Set of TEP for decision making. The proposed differentiation based TEP framework is able to determine the critical transmission components for constituting the minimum backbone grid, which is of significant reference value to the TEP under the meteorological disaster-prone environment. 


\section{References}

[1] Maghouli P, Hosseini SH, Buygi MO, Shahidehpour M. A multi-objective framework for transmission expansion planning in deregulated environments. IEEE Trans. Power Syst.; 2009; 24(2):1051-61.

[2] Molina JD, Rudnick, H. Transmission expansion plan: Ordinal and metaheuristic multiobjective optimization. In: Proc. of 2011IEEE Trondheim PowerTech., 2011:1-6.

[3] Alseddiqui J, Thomas RJ. Transmission expansion planning using multi-objective optimization. In: Proc. of 2006 IEEE Power Eng. Soc. Gen. Meet, 2006:1-6.

[4] Latorre G, Darío CR, Areiza JM, Villegas A. Classification of publications and models on transmission expansion planning. IEEE Trans. Power Syst.; 2003; 18(2):938-46.

[5] Tessema BT. Strategies for electrical network planning. MS thesis, Delft University of Technology, 2011.

[6] Cheng H-Z ,Gao C-W,Ma Z-L, Zhu Z-L, Xu J, Wang X-H. The lexicographically stratified method for multi-object optimal electric power network planning. Proceedings of the CSEE; 2003; 23(10):11-6.

[7] Cai L, Zhang Y, Cheng H. Transmission network planning incorporating transient stability. Autom. Electr. Power Syst., 2001; 25(6):45-8.

[8] Risk Management Solutions, Inc. The 1998 ice storm: 10-year retrospective. RMS Special Report, 2008.

[9] Q Chen, Yin X, You D, et al. Review on blackout process in China southern area main power grid in 2008 snow disaster. In: Proc. of 2009 PES General Meeting, 2009:1-8.

[10] Cheng H, Gao C, Ma Z, et al. General optimized model of multi-objective electric power network planning. Journal of Shanghai Jiaotong University, 2004; 38(8):1229-32.

[11] Sun H, Yu DC. A multiple-objective optimization model of transmission enhancement planning for independent transmission company (ITC). In: Proc. of IEEE 2000 Power Engineering Society Summer Meeting, 2000:2033-8.

[12] Qu G, Cheng H, Yao L, et al. Transmission surplus capacity based power transmission expansion planning. Electric Power Research, 2010; 80(1):19-27.

[13] Ma C, Xue Y, Lu T, et al. A review of transmission planning methods. Autom. Electr. Power Syst., 2006; 30(12):97-101.

[14] Chen L, Zheng Y, et al. Transmission network planning with FACTS devices based on modified adaptive genetic algorithm. Proceedings of the CSEE, 1999; 19(1):6-9.

[15] Ji X, Wang C. Transmission dynamic expansion considering demand elasticity. Proceedings of the CSEE, 2002; 22(11):23-27.

[16] Jin H, Cheng H, Yang X, et al. Application of fuzzy set pair analysis in multi-objective transmission network planning considering ATC. Electr. Power Syst., 2005; 29(21):45-49.

[17] Mori H, Kakuta H. A CNSGA-II based method for multi-objective probabilistic transmission network expansion planning. In: Proc. of IEEE PES Gen. Meet. 2010:1-6.

[18] Quinn MP, Izzuddin BA. Global structural cost optimization by simulated annealing. Advances in Engineering Computational Technology,1998:143-7.

[19] Jin Y, Cheng H, et al. Improved particle swarm optimization method and its application in power transmission network planning. Proceedings of the CSEE, 2005; 25(4):45-50.

[20] Wen F, Chang CS. Transmission network optimal planning using the Tabu search method. Electr Power Syst Res, 1997; 42(2):153-63.

[21] Cheng X, Ye X, Liang Y, et al. Improved Ant algorithm for transmission network expansion planning. Autom. Electr. Power Syst., 2006; 30(20):37-40.

[22] Zhang Y, Yang H, et al. The stochastic optimal dispatch model considering the uncertain line failures events under extreme weather disasters. In: Proc. of POWERCON, 2010:1-6.

[23] Terms for Reporting and Analysing Outages of Electrical Transmission and Distribution Facilities and Interruptions To Customers Services. IEEE Standard 346; 1973

[24] Billinton R, Singh G. Application of adverse and extreme adverse weather: modelling in transmission and distribution system reliability evaluation. IEE Proc., Gener. Transm. Distrib., 2006; 153(1):115-20. 